\title{
Link Scheduling in Multi-Transmit-Receive Wireless Networks
}

\author{
Hong-Ning Dai \\ Macau University of Science and Technology, Macau \\ hndai@ieee.org
}

\author{
Soung Chang Liew and Liqun Fu \\ The Chinese University of Hong Kong, Hong Kong \\ \{soung,lqfu6\}@ie.cuhk.edu.hk
}

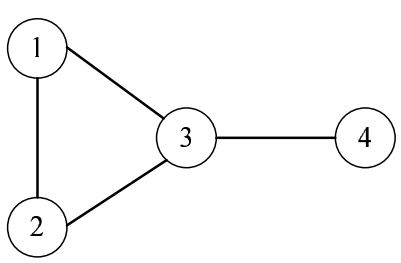

\begin{abstract}
This paper investigates the problem of link scheduling to meet traffic demands with minimum airtime in a multitransmit-receive (MTR) wireless network. MTR networks are a new class of networks, in which each node can simultaneously transmit to a number of other nodes, or simultaneously receive from a number of other nodes. The MTR capability can be enabled by the use of multiple directional antennas or multiple channels. Potentially, MTR can boost the network capacity significantly. However, link scheduling that makes full use of the MTR capability must be in place before this can happen. We show that optimal link scheduling can be formulated as a linear program (LP). However, the problem is NP-hard because we need to find all the maximal independent sets in a graph first before the LP can be set up. We propose two computationally efficient algorithms, called Heavy-Weight-First (HWF) and Max-DegreeFirst (MDF) to solve this problem. Simulation results show that both HWF and MDF can achieve superior performance in terms of runtime and optimality. Specifically, we have conducted 1,000 simulation experiments with different network topologies and traffic demands. On average, the HWF and MDF solutions are within $90 \%$ of the optimal solutions.
\end{abstract}

\section{INTRODUCTION}

This paper concerns the problem of link scheduling to minimize airtime usage in a new class of wireless networks called multi-transmit-receive (MTR) wireless networks. In an MTR network, a node can simultaneously transmit to a number of other nodes, or simultaneously receive from a number of other nodes. However, a node cannot simultaneously transmit and receive (i.e., the half-duplexity is still in place). Enabling this capability of MTR networks is the use of multiple directional antennas at a node [1]-[5] or the use of multiple channels on multiple collocated radios at a node [6]. Potentially, this capability can increase the network capacity significantly [2], [3], [6]. Details about MTR networks will be presented in Section II

Potentially, MTR networks can schedule more wireless links than conventional wireless networks. Take Fig. 1 as an example. In this four-node network, the mutually connected nodes 1 and 2 are connected with nodes 3, which is in turn connected with node 4 . Since there are four edges, there are totally eight directional links. Denote the link set by $\{(1,2),(1,3),(2,1),(2,3),(3,1),(3,2),(3,4),(4,3)\}$. For a conventional network, where multiple simultaneous transmissions or receptions at a node are not allowed, at most two links can be active at a given time (e.g., $(1,2)$ and $(4,3)$ ). However, an MTR network allows three links to be active simultaneously (e.g., $(1,2),(1,3),(4,3))$.

This paper considers the link-scheduling problem of determining the minimum Time Division Multiple Access (TDMA) frame length while fulfilling the traffic demands in MTR networks. Although there have been prior studies on MTR networks [1]-[5], [7]-[10], this particular link scheduling problem (which is a well studied classical problem under the context of non-MTR networks [11]-[16]) has not been investigated as far as we know. Previously proposed MTR MAC protocols, such as 2P [3], WiLDNet [2] and JazzyMAC [5], are not efficient in that a node needs to maintain all of its links in transmit mode for the same time duration regardless of the actual link traffic demands. The links with lower traffic demands will sit idle while the other links of the node transmit; meanwhile, the nodes at the other ends of the idle links are not allowed to transmit - this is purely a constraint imposed by the MAC rather than an MTR constraint. Consider the four-node example in Fig. 1 again. Suppose a traffic demand for the above link set is $[1,1,1,1,1,1,2,1]$. Then, 2P, WiLDNet and JazzyMAC obtain a sub-optimal schedule, $\{(1,2),(1,3),(4,3)\},\{(2,1),(2,3)\},\{(3,4)\},\{(3,1),(3,2)$, $(3,4)\}$, which requires four time slots. However, only three time slots are required for an optimal schedule: $\{(2,1),(3,1),(3,4)\},\{(1,2),(3,2),(3,4)\},\{(1,3),(2,3)$, $(4,3)\}$.

The primary research contributions of our paper are summarized as follows.

1. We provide a formal specification of an MTR network, and formulate the link-scheduling problem of determining the minimum frame length required to meet the underlying link-traffic demands.

2. We show that solving the link scheduling problem optimally is NP-hard, since we need find all the maximal independent sets (MIS) in a graph. 
3. We propose two computationally efficient heuristic algorithms to tackle this problem. The first algorithm is a Heavy-Weight-First (HWF) algorithm, which gives priority to the links with the heaviest traffic demands in its schedule. The second algorithm is a MAX-Degree-First (MDF) algorithm, which gives priority to the links with the maximum degree in a conflict graph in its schedule.

4. We conduct extensive simulations based on regular and random network topologies, with symmetric and asymmetric traffic demands. The simulation results show that both HWF and MDF can typically obtain solutions within $90 \%$ of the optimal solutions over 1,000 simulation experiments based on symmetric and asymmetric traffic demands.

The rest of the paper is organized as follows. In Section $\Pi$ we present the network model, basic assumptions and problem formulation. Section III presents two heuristic algorithms. We show the simulation results in Section IV Section $\nabla$ concludes the paper.

\section{Network Model and Problem Formulation}

In this section, we specify the MTR network model formally. We then formulate the link-scheduling problem of finding the minimum frame length in MTR networks as a linear program (LP). The previously proposed MTR MAC protocols (e.g., 2P [3], WiLDNet [2] and JazzyMAC [5]) are shown to be sub-optimal.

A Multi-Transmit-Receive (MTR) network is defined as follows.

Definition 1: In an MTR network, each node has a set of neighbor nodes with whom it forms links. At any given time,

R1. A node can transmit simultaneously on a subset of its outgoing links.

R2. A node can receive simultaneously on a subset of its incoming links.

R3. A node cannot do operations R1 and R2 simultaneously, (i.e., a node cannot transmit and receive simultaneously).

Given an MTR network, we are interested in how to minimize the TDMA slots required to meet the underlying link traffic demands.

\section{A. Centralized Scheduling Problem}

Let the link traffics be specified by the traffic matrix $T=\left[t_{i j}\right]$, where $t_{i j}$ is the amount of traffic from node $i$ to its neighboring node $j$. At any given time, let the set of active links in the network be indicated by an indicator matrix, $M^{(k)}=\left[m_{i j}^{(k)}\right]$, where $m_{i j}^{(k)}=1$ if link $(i, j)$ is active, and $m_{i j}^{(k)}=0$ if link $(i, j)$ is inactive.

Definition 2: An indicator matrix is called a matching matrix if all nodes conform to rules R1, R2 and R3.

Let us consider the four-node network as shown in Fig. 1 . In this network, an example of a matching matrix is:

$$
M^{(1)}=\left(\begin{array}{cccc}
0 & 1 & 1 & 0 \\
0 & 0 & 0 & 0 \\
0 & 0 & 0 & 0 \\
0 & 0 & 1 & 0
\end{array}\right)
$$

where matrix $M^{(1)}$ indicates that node 1 simultaneously transmits to nodes 2 and 3 while node 4 transmits to node 3 . Note that the matching matrix $M^{(1)}$ is maximal in that you cannot turn any of its 0 elements to 1 without violating R3.

Definition 3: A matching matrix is maximal if none of its 0 elements can be turned to 1 (while maintaining all its 1 elements at 1) without violating the rules in definition 1.

When we consider the link scheduling, we only need to consider the maximal matching matrix. Suppose there be $K$ maximal matching matrices. Then, the problem that we are considering is as follows:

$$
\begin{array}{cc}
\min & \sum_{k=1}^{K} x_{k} \\
\text { s.t. } & \sum_{k=1}^{K} M^{(k)} x_{k} \geq T \\
& x_{k} \geq 0 \text { for all } k
\end{array}
$$

where $x_{k}$ denotes the number of time slots allocated to maximal matching matrix $M^{(k)}$.

\section{B. Sub-optimal Scheduling}

In previously proposed MAC protocols for MTR networks networks, such as 2P [3], WiLDNet [2] and JazzyMAC [5], each node alternates between two phases (2P): simultaneous reception (SynRx) and simultaneous transmission (SynTx). In addition, a node is required to maintain all of its links in transmit mode for the same time duration regardless of the link traffic demands, resulting in inefficiency. In particular, the simultaneous synchronized operations in these MAC protocols indicate that when a node transmits, none of its neighbor nodes can transmit. As far as scheduling is concerned, this constraint is equivalent to (virtually) turning R1 in Definition 1 to a more restrictive requirement, as follows:

$\mathrm{R} 1^{\prime}$ When a node transmits, it transmits on all its outgoing links.

Constraint $\mathrm{R} 1^{\prime}$ plus the half-duplexity in constraint R3 implies that the neighbors of a node cannot transmit at the same time.

Since with $\mathrm{R} 1^{\prime}$, when a node $i$ transmits, it transmits on all outgoing links, we might as well replace the traffic requirements for outgoing traffic from node $i,\left(t_{i 1}, t_{i 2}, \ldots, t_{i N}\right)$, by one single number, $t_{i}=\max _{j} t_{i j}$. Then $t=\left(t_{i}\right)$ is the traffic vector describing the transmission requirements of all nodes.

Let $S^{(l)}=\left(s_{i}^{(l)}\right)$ be a column indicator vector in which $s_{i}^{(l)}=1$ if node $i$ transmits and $s_{i}^{(l)}=0$ if node $i$ does not transmit. With respect to the graph describing the network, $S^{(l)}$ is basically an independent se 1 if it is to conform to R1', $\mathrm{R} 2$, and R3. It suffices to consider the maximal independent set (MIS) 2 in our scheduling problem. Suppose that there are $L$ MIS. Then, the scheduling problem can be formulated as

\footnotetext{
${ }^{1} \mathrm{An}$ independent set is a subset of vertices such that no edge joins any two of them.

${ }^{2} \mathrm{~A}$ maximal independent set is an independent set that is not the subset of another independent set.
} 
follows:

$$
\begin{array}{cc}
\min & \sum_{l=1}^{L} x_{l} \\
\text { s.t. } & \sum_{l=1}^{L} S^{(l)} x_{l} \geq t \\
& x_{l} \geq 0 \text { for all } l
\end{array}
$$

Since Eq. (2) is defined in a more restrictive way, the solution to Eq. (1) cannot be worse than that of Eq. (2).

Consider the four-node example (Fig. 1) again. In addition, suppose we have the following traffic requirements:

$$
T=\left(\begin{array}{llll}
0 & 1 & 1 & 0 \\
1 & 0 & 1 & 0 \\
1 & 1 & 0 & 2 \\
0 & 0 & 1 & 0
\end{array}\right)
$$

Then, we replace the outgoing traffic requirements of each node by a single number and get a vector:

$$
t=[1,1,2,1]^{T}
$$

In the four-node example, there are three maximal independent sets (MIS):

$$
\begin{aligned}
S^{(1)} & =[1,0,0,1]^{T} \\
S^{(2)} & =[0,1,0,1]^{T} \\
S^{(3)} & =[0,0,1,0]^{T}
\end{aligned}
$$

We can verify that the optimal solution to the problem defined in Eq. (2) is given by

$$
x_{1}=1, \quad x_{2}=1, \quad x_{3}=2
$$

Thus, a total of four units of airtime is needed.

Now, let us go back to the original problem defined in Eq. (1). First of all, the maximal matching matrices corresponding to the above MIS are:

$$
\begin{aligned}
& M^{(1)}=\left(\begin{array}{llll}
0 & 1 & 1 & 0 \\
0 & 0 & 0 & 0 \\
0 & 0 & 0 & 0 \\
0 & 0 & 1 & 0
\end{array}\right) \\
& M^{(2)}=\left(\begin{array}{llll}
0 & 0 & 0 & 0 \\
1 & 0 & 1 & 0 \\
0 & 0 & 0 & 0 \\
0 & 0 & 1 & 0
\end{array}\right) \\
& M^{(3)}=\left(\begin{array}{llll}
0 & 0 & 0 & 0 \\
1 & 0 & 0 & 0 \\
1 & 1 & 0 & 1 \\
0 & 0 & 0 & 0
\end{array}\right)
\end{aligned}
$$

In addition, there are three additional maximal matching matrices, shown as follows:

$$
M^{(4)}=\left(\begin{array}{cccc}
0 & 0 & 0 & 0 \\
1 & 0 & 0 & 0 \\
1 & 0 & 0 & 1 \\
0 & 0 & 0 & 0
\end{array}\right)
$$

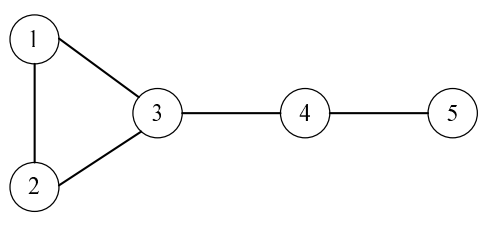

Fig. 2. A five-node network

$$
\begin{aligned}
& M^{(5)}=\left(\begin{array}{llll}
0 & 1 & 0 & 0 \\
0 & 0 & 0 & 0 \\
0 & 1 & 0 & 1 \\
0 & 0 & 0 & 0
\end{array}\right) \\
& M^{(6)}=\left(\begin{array}{llll}
0 & 0 & 1 & 0 \\
0 & 0 & 1 & 0 \\
0 & 0 & 0 & 0 \\
0 & 0 & 1 & 0
\end{array}\right)
\end{aligned}
$$

Indeed, the optimal solution to Eq. (1) is given by assigning airtimes to the matrices in Eq. (4) only:

$$
x_{1}=x_{2}=x_{3}=0, x_{4}=1, x_{5}=1, x_{6}=1
$$

This solution requires three units of airtime, which is less than four units in Eq. (2).

The above example gives rise to an interesting observation: the matrices in Eq. (4) are the transposes of the matrices in Eq. (3). Thus, we have the following theorem,

Theorem 1: If $M^{(k)}$ conforms to constraints R1, R2, and R3 as defined in Definition 11 then so does its transpose.

Proof: It is obvious that constraints R1 and R2 are still fulfilled in the transposed matching matrix. The direction of transmission on a link simply gets reverse in the transposed matching matrix, so that the transmitters become the receivers, and vice versa. Thus, the half-duplexity constraint, R3 is still fulfilled.

In the four-node example above, the set of maximal matching matrices in Eq. (1) can be found from the MIS in Eq. (2). More specifically, each MIS in Eq. (2) leads to two maximal matching matrices. In one matrix, each vertex in MIS is a transmit node and it transmits on all its outgoing links; in the other one, each vertex in MIS is a receive node and it receives on all its incoming links. Thus, each MIS induces two maximal matching matrices.

A question then is whether all maximal matching matrices are induced from an MIS. Unfortunately, the answer is no. Consider the following five-node network, as shown in Fig. 2 . A possible maximal matching matrix to this network is shown as follows:

$$
M^{\prime}=\left(\begin{array}{lllll}
0 & 0 & 1 & 0 & 0 \\
0 & 0 & 1 & 0 & 0 \\
0 & 0 & 0 & 0 & 0 \\
0 & 0 & 0 & 0 & 0 \\
0 & 0 & 0 & 1 & 0
\end{array}\right)
$$

In this matching, nodes 1,2 , and 5 are transmitters; and node 3 and 4 are receivers. The transmitters do not form an MIS because nodes 1 and 2 are neighbors. The receivers do 


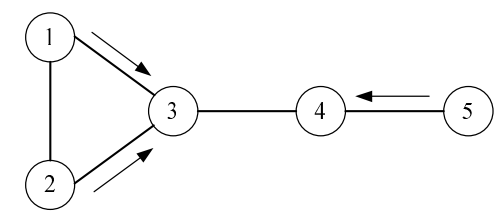

Fig. 3. The active links in the five-node network

not form an MIS because nodes 3 and 4 are neighbors. Fig. 3 depicts the active links in maximal matching matrix $M^{\prime}$.

In general, the number of maximal matchings can be more than twice the number of MIS. Then, how are maximal matching matrices related to MIS? To establish the relation, we need to model the network with a different graph. We use a conflict graph to describe the relationship between two conflicting links. In this graph, each directional link is denoted by a vertex, and there is an edge between two vertices if the two associated links cannot be active at the same time. The conflict graph for the five-node network above is shown as Fig. 4, where vertex $(i, j)$ represents link $l_{i j}$. With the modified graph, we can then formulate the problem in Eq. (1). In the traffic vector, $t_{v}=t_{i j}$ where $v$ represents the vertex corresponding to link $l_{i j}$.

\section{Problem Restatement}

In optimization problem defined in Eq. (1), we represent a matching by a matrix $M^{(k)}$ for pedagogical purposes. We now define a more economical representation.

Definition 4: A matching $A$ in an MTR network is a subset of links that conform to R1, R2 and R3.

Definition 5: A matching is said to be maximal if it is not contained in any other matching.

Let $E=\left\{E_{j}: 1 \leq j \leq|E|\right\}$ be the set of all the feasible matchings. The number of time slots allocated to each feasible matching $E_{j}$ is denoted by a non-negative variable $u_{j}$.

Let $N$ be the total number of links in the network. We introduce an $N \times|E|$ incidence matrix $Q$ with elements $q_{i j}$ such that

$$
q_{i j}= \begin{cases}1, & \text { if link } i \text { is in matching } E_{j}, \\ 0, & \text { otherwise. }\end{cases}
$$

where each column in $Q$ indicates the links in a matching.

We also convert the traffic matrix $T=\left[T_{i j}\right]$ to a vector $\mathbf{f}=\left(f_{i j}\right)^{T}$, where $f_{i j}=T_{i j}$ for $i, j$ such that $T_{i j} \neq 0$. Then, the problem defined in Eq. (1) can be casted as a linear program as follows:

$$
\begin{array}{cc}
\min & \mathbf{e}^{T} \mathbf{u} \\
\text { s.t. } & Q \cdot \mathbf{u} \geq \mathbf{f} \\
& \mathbf{u} \geq 0 \text { for all } k
\end{array}
$$

where $\mathbf{e}$ is a vector whose components are all 1 's, $\mathbf{f}=$ $\left(f_{1}, f_{2}, \ldots, f_{N}\right)^{T}$ and $\mathbf{u}=\left(u_{1}, u_{2}, \ldots, u_{|E|}\right)^{T}$.

The difficulty of the above problem lies in how to find all matchings $Q$ (equivalent to finding all the independent sets in the associated conflict graph, which is NP-complete).

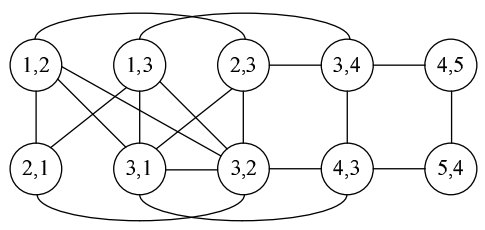

Fig. 4. The conflict graph for the five-node network

This motivates us to investigate heuristic algorithms to solve this problem. We will present two computationally efficient algorithms in the next section.

\section{Heuristic AlgorithmS}

In this section, we propose two heuristic algorithms, heavyweight-first (HWF) algorithm and max-degree-first (MDF) algorithm to solve the link-scheduling problem defined in Eq. (5). HWF is a greedy algorithm that always chooses links with the maximum traffic demand (the heaviest weight) into the scheduling set during each round until all the traffic is satisfied. MDF, on the other hand, chooses links with the maximum degree in the conflict graph during each round.

Both HWF and MDF make use of a conflict graph to capture constraints $\mathrm{R} 1, \mathrm{R} 2$ and $\mathrm{R} 3$. In the conflict graph, a link $(i, j)$ is represented by a vertex. Two links, $(i, j)$ and $(k, l)$, conflict with each other if and only if $i=l$ or $j=k$. An edge is drawn between the vertices representing $(i, j)$ and $(k, l)$ if they conflict with each other.

\section{A. Heavy-Weight-First Algorithm}

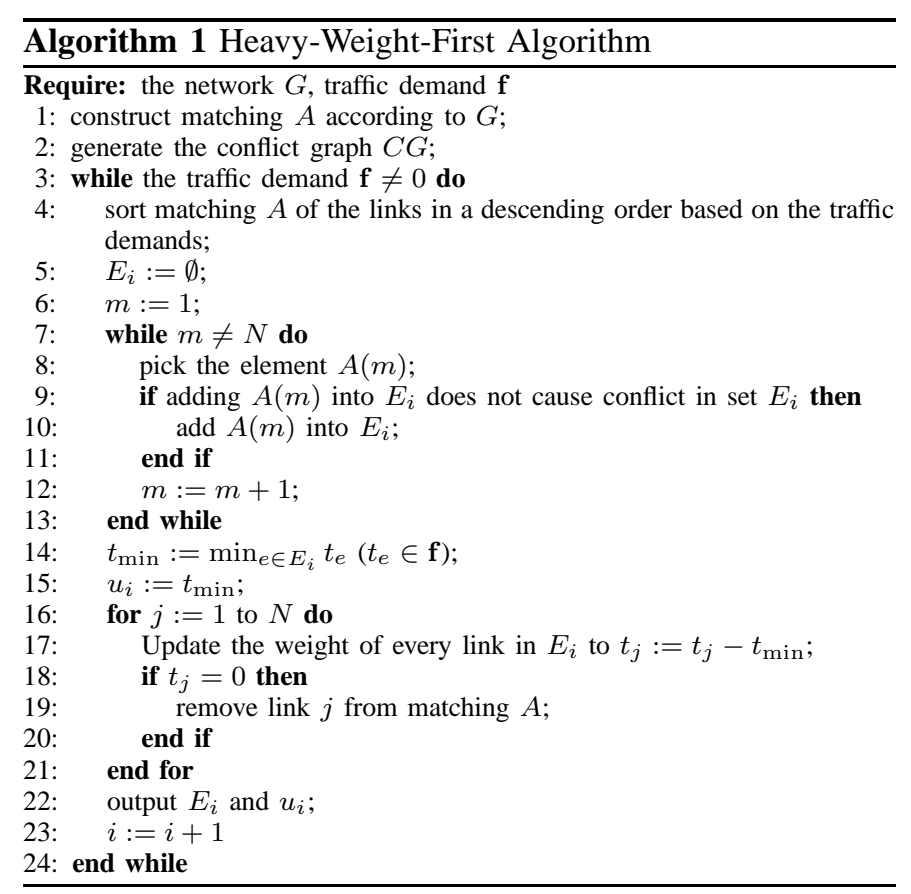

In Heavy-Weight-First algorithm (HWF), we first sort the links according to their traffic demands in a descending order. To construct a matching, $E_{i}$, we go through the link one by one. A link will be included into $E_{i}$ if it does not conflict with 


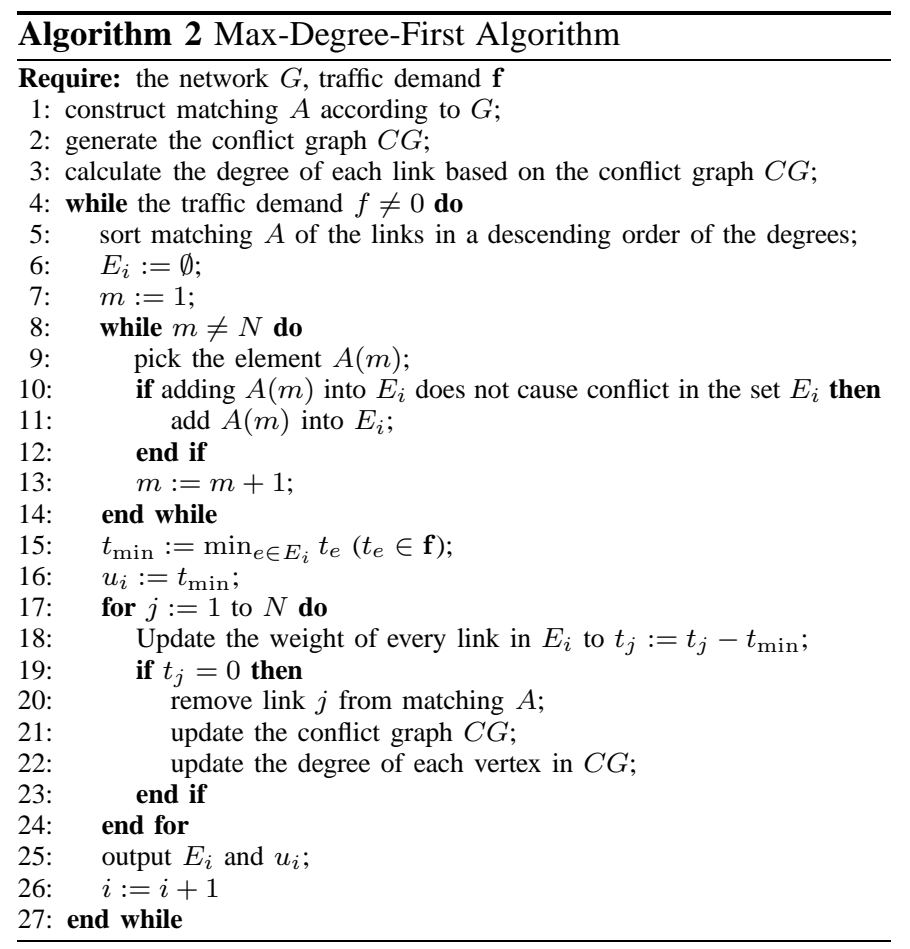

the existing links in $E_{i}$ according to the conflict graph. Once we have gone through all the links in the sorted list, we then identify the link in $E_{i}$ with the least amount of traffic. Let us say this is link $k$, with traffic $f_{k}$. We then assign $f_{k}$ time slots to matching $E_{i}$. We subtract $f_{k}$ from the traffic of all the links in $E_{i}$, and remove link $k$ and other links in $E_{i}$ with the same amount of traffic (if any) from further consideration: there is not traffic left to be scheduled for these links. The links are then resorted according to their remaining traffics. The above process is iterated until all traffic demands are met. At most $N$ iterations are needed, since each iteration removes at least one link from further consideration.

\section{B. Max-Degree-First Algorithm}

In MDF, we sort the links according to their degrees in the conflict graph in a descending order. Other than the different way of sorting the links, the algorithm of MDF is essentially the same as that of HWF. In particular, at least one link will be removed at the end of each iteration. The degrees of the neighbors to this link will be updated. The remaining links will also need to be resorted accordingly before the next iteration. During each iteration, at least one link will be removed. Thus, similar to HWF, MDF needs at most $N$ iterations.

\section{Simulation Results}

We have conducted extensive simulation experiments on a Pentium $2.86 \mathrm{GHz}$ PC with 2GB memory. We consider several types of networks: (1) regular networks, including the linear network in Fig. 5, the grid network in Fig. 6, the ring network in Fig. 7 and the fully-connected network in Fig. 8, (2) random networks with varying degrees of connectivity. We

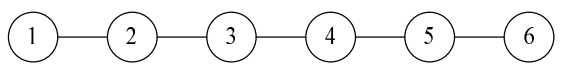

Fig. 5. The linear network

also consider various traffic demands, symmetric as well as asymmetric.

Symmetric traffic demands mean that $f_{i j}=f_{j i}$ for all pairs of $i$ - $j$ neighbors. Symmetric traffic demands, on the other hand, mean that $f_{i j} \neq f_{j i}$ for some $i$ - $j$ neighbors.

When the network topology is bipartite, we can easily find the optimal solution for the link scheduling problem. Details about this issue are presented in Appendix A. Note that since a tree network topology can always be organized into a bipartite graph, link scheduling in an MTR tree network is also easy.

\section{A. Performance in Regular Networks}

For the investigation of regular networks, we first present the simulation results of the linear network in Fig. 5. For this small network, we can easily find the optimal solution by simple hand calculation.

We present the simulation results in Table I The link set is $\{(1,2),(2,1),(2,3),(3,2),(3,4),(4,3),(4,5),(5,4),(5,6),(6,5)\}$. Both HWF and MDF achieve the optimal solutions for both symmetric traffic demands (simulation No. 1 and simulation No. 2) and asymmetric traffic demands (simulation No. 3).

TABLE I

SIMULATION RESULTS FOR THE LINEAR NETWORK

\begin{tabular}{c|c|c|c|c}
\hline No. & Traffic demands f & HWF & MDF & optimal \\
\hline \hline 1 & {$[5,5,5,5,5,5,5,5,5,5]$} & 10 & 10 & 10 \\
\hline 2 & {$[6,6,4,4,8,8,5,5,7,7]$} & 16 & 16 & 16 \\
\hline 3 & {$[6,3,4,5,7,8,5,2,7,9]$} & 16 & 16 & 16 \\
\hline
\end{tabular}

In the second set of simulations, we consider a grid network with nine nodes, as shown in Fig. 6 The simulations results are shown in Table. III The link set is $\{(1,2),(1,6),(2,1),(2,3),(2,5),(3,2),(3,4),(4,3),(4,5$ )$,(4,9),(5,2),(5,4),(5,6),(5,8),(6,1),(6,5),(6,7),(7,6$ )$,(7,8),(8,5),(8,7),(8,9),(9,4),(9,8)\}$. The simulation results show that both HWF and MDF obtain the optimal solution of 10 for the symmetric traffic demands (simulation No. 1). But for asymmetric traffic demands (simulation No. 2 ), HWF obtains a solution of 20 , which is greater than the optimal solution of 18 ; while MDF obtains the optimal solution of 18 .

TABLE II

SIMULATION RESULTS FOR THE GRID NETWORK

\begin{tabular}{c|c|c|c|c}
\hline No. & Traffic demand f & HWF & MDF & optimal \\
\hline \hline 1 & {$[5,5,5,5,5,5,5,5,5,5,5,5$,} & 10 & 10 & 10 \\
& $5,5,5,5,5,5,5,5,5,5,5,5]$ & & & \\
\hline 2 & {$[7,8,8,4,7,2,8,1,3,1,1,9$,} & 20 & 18 & 18 \\
& $7,4,10,1,5,4,8,8,2,5,5,7]$ & & & \\
\hline
\end{tabular}

The third set of simulations are based on a ring network with six nodes, as shown in Fig. 7. Simulations results are shown in Table III The link set is 


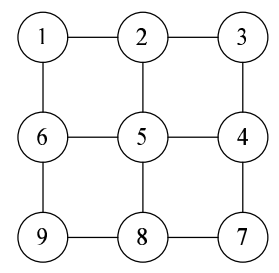

Fig. 6. The grid network

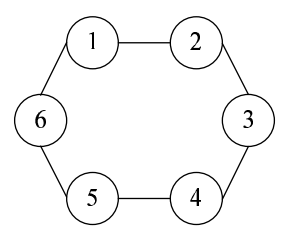

Fig. 7. The ring network

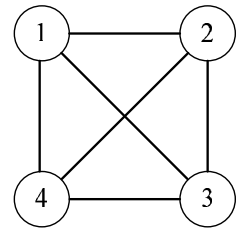

Fig. 8. The fullyconnected network
$\{(1,2),(1,6),(2,1),(2,3),(3,2),(3,4),(4,3),(4,5),(5,4)$ $,(5,6),(6,1),(6,5)\}$. The simulation results show that both HWF and MDF achieve the optimal solution of 10 for symmetric traffic demands (simulation No. 1) and asymmetric traffic demands (simulation No. 2).

TABLE III

SIMULATION RESULTS FOR THE RING NETWORK

\begin{tabular}{c|c|c|c|c}
\hline No. & Traffic demands f & HWF & MDF & optimal \\
\hline \hline 1 & {$[5,5,5,5,5,5,5,5,5,5,5,5]$} & 10 & 10 & 10 \\
\hline 2 & {$[2,5,10,3,4,6,7,8,9,11,4,12]$} & 23 & 23 & 23 \\
\hline
\end{tabular}

We have also conducted 1,000 simulation experiments (with different traffic demands) for each of the following networks: the linear network (Fig. 5), the grid network (Fig. 6), the ring network (Fig. 7) and the fully-connected network (Fig. 8).

In order to compare the solutions obtained by the proposed algorithms with optimal solutions, we introduce the percentage cost penalty [12] as a performance measure. Its definition is as follows:

$$
P=\frac{T-T_{o p t}}{T_{\text {opt }}} \times 100 \%
$$

where $T$ denotes the total number of time slots obtained by the heuristic algorithm and $T_{o p t}$ is the total number of time slots in the optimal solution.

We compute $P$ of HWF and MDF over the 1,000 experiments and present the averaged $P$ values in Table 4 . In each experiment, we generate a random traffic demand vector $\mathbf{f}$, where each element of $\mathbf{f}$ conforms to a discrete uniform distribution with values ranging from 1 to 10 .

The results in Table IV show that MDF outperforms HWF in the linear network, the grid network and the ring network. But HWF performs better in the fully-connected network. The above results can be explained intuitively as follows. Recall that a link is removed at the end of each iteration in MDF or HWF. The nature of MDF is such that the link being removed has a high degree in the conflict graph. In this sense, MDF tends to remove many edges in the conflict graph. As a result, in a sparsely connected network (e.g., the linear network in Fig. 5, the grid network in Fig. 6 and the ring network in Fig. 7), many links become conflict-free after several iterations.

By contrast, in a densely connected network, (e.g., the fully-connected network in Fig. 8), the links are so closely connected together in the conflict graph (e.g., in Fig. 9) that very few links can become conflict-free even after several iterations. As to why HWF tends to have better performance

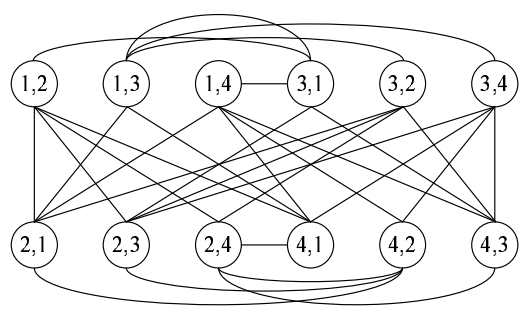

Fig. 9. The conflict graph for the fully-connected network

than MDF when the network is densely connected, we shall defer the intuitive explanation to Section 4.2, where we focus on large-scale random networks.

TABLE IV

SIMULATION RESULTS FOR RANDOM TRAFFIC IN VARIED REGULAR NETWORKS

\begin{tabular}{c|c|c}
\hline & $\begin{array}{c}\text { Average cost penalty } \\
\text { of HWF }\end{array}$ & $\begin{array}{c}\text { Average cost penalty } \\
\text { of MDF }\end{array}$ \\
\hline \hline $\begin{array}{c}\text { Linear network (Fig. } \\
5\end{array}$ & $5.49 \%$ & $0 \%$ \\
\hline Grid network (Fig. 6) & $8.16 \%$ & $0 \%$ \\
\hline Ring network (Fig. 7) & $7.97 \%$ & $0 \%$ \\
\hline $\begin{array}{c}\text { Fully-connected } \\
\text { network (Fig. 8) }\end{array}$ & $4.04 \%$ & $9.15 \%$ \\
\hline
\end{tabular}

\section{B. Performance in Random Networks}

For comparison purposes, we carry out exhaustive search to find optimal solutions. We compare the average runtime of HWF and MDF with that of exhaustive search. To reduce the runtime of exhaustive search, we use a branch-and-bound algorithm, first proposed in [17].

We generate random network topologies, represented by random matrix $G$, which is symmetric with zero diagonal. In $G$, entry $g_{i j}=1$ if there is a pair directional links between nodes $i$ and $j$; and $g_{i j}=0$ otherwise. For our simulation experiments, $\operatorname{Pr}\left[g_{i j}=1\right]=p=0.5, \forall i, j$. Thus, the networks being simulated are densely connected in that a node is connected to half of the other nodes on average. The number of nodes in the simulated networks is $n=6$. Thus, the maximum number of unidirectional links is $n(n-1)=30$. Each directional link will conflict with at most $2 n-1$ other links under the MTR constraints. Thus, in the associated conflict graph, each link has a degree ranging from 1 to 11 when $n=6$.

In the first set of simulations, we consider symmetric traffic demands. If there is a link between node $i$ and node $j$ (i.e., $g_{i j}=1$ ), then the traffic between them, $f_{i j}=f_{j i}$, is randomly generated according to the discrete uniform distribution with values ranging from 1 to 10 . We conduct 1,000 experiments and present the results in Fig. 10 and Table $\mathrm{V}$. Each experiment is based on one random network $G$ and one associated 


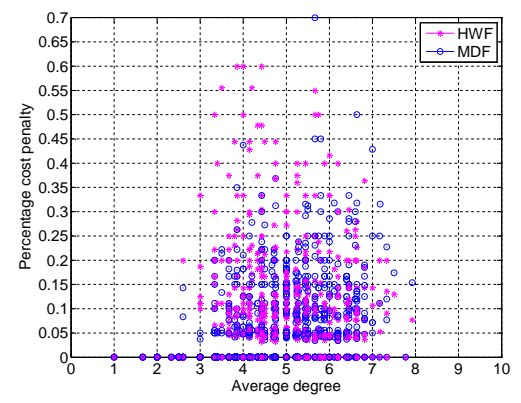

Fig. 10. The percentage cost penalty: symmetric traffic

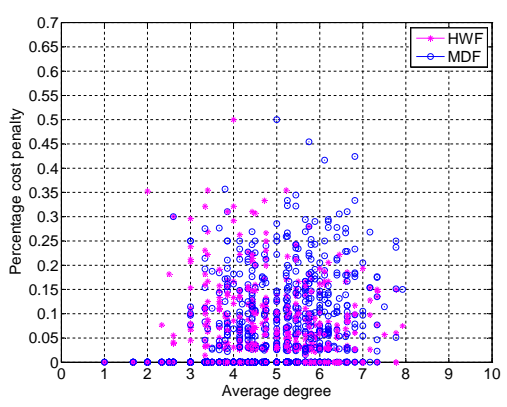

Fig. 11. The percentage cost penalty: asymmetric traffic

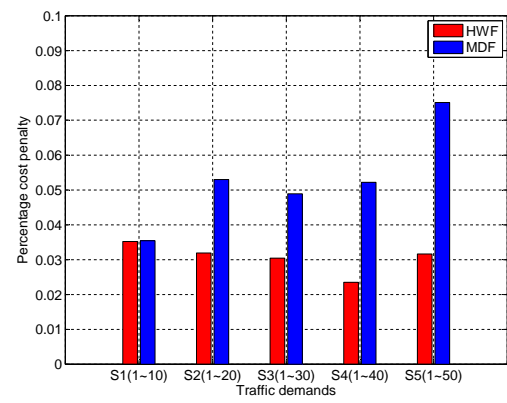

Fig. 12. The percentage cost penalty vs traffic demands
TABLE V

SIMULATION RESULTS FOR SYMMETRIC TRAFFIC OVER RANDOM NETWORKS

\begin{tabular}{c|c|c|c|c}
\hline & $\begin{array}{c}\text { No. of obtained } \\
\text { solutions within } \\
100 \% \text { optimality }\end{array}$ & $\begin{array}{c}\text { No. of obtained } \\
\text { solutions with } \\
P \text { within } 10 \%\end{array}$ & $\begin{array}{c}\text { Average } \\
P\end{array}$ & $\begin{array}{c}\text { Average } \\
\text { runtime } \\
\text { (second) }\end{array}$ \\
\hline \hline $\begin{array}{c}\text { Exhaustive } \\
\text { Search }\end{array}$ & 1,000 & 1,000 & $0 \%$ & 1.7522 \\
\hline HWF & 540 & 781 & $6.40 \%$ & 0.0015 \\
\hline MDF & 549 & 786 & $5.59 \%$ & 0.0017 \\
\hline
\end{tabular}

random demand f. Fig. 10 plots percentage cost penalty versus average link degree. Table $\nabla$ gives the statistics of the 1,000 experiments.

Fig. 10 and Table $\nabla$ show that both HWF and MDF achieve reasonably good performance. In particular, Table $\mathrm{V}$ shows that there are nearly 800 solutions obtained by HWF and MDF with penalty cost no greater than $10 \%$. On average, HWF and MDF have average $P$ of $6.40 \%$ and $5.59 \%$, respectively. Table $\mathrm{V}$ also shows that the average runtime of the two algorithms is much smaller than that of exhaustive search.

We have also conducted 1,000 simulations based on asymmetric traffic demands. The simulation results are presented in Fig. 11 and Table VI] The traffic demand $f_{i j}$ of each link $(i, j)$ is randomly generated according to the discrete uniform distribution with values ranging from 1 to 10 . But the traffic in the opposite direction, $f_{j i}$ is not set to $f_{i j}$; rather, it is generated anew using the same distribution. It is shown in Fig. 11 and Table VI that HWF outperforms MDF in this asymmetric traffic scenario. In particular, Table $\mathrm{VI}$ shows that HWF obtain 872 solutions with $P$ less than $10 \%$ versus 779 obtained by MDF. On average, HWF has a lower average $P$ of $3.42 \%$ versus $5.32 \%$ of MDF.

HWF outperforms MDF in the asymmetric case because HWF can "compact traffic demands" as it runs. By compacting traffic demands, we mean HWF can decrease the range of the traffic demands in the network after each iteration. To see this, suppose we have a traffic demand, $\mathbf{f}=[1,2,3,4,5,6,7,8,9,10]$, where the traffic ranges from 1 to 10 . Suppose that in the first iteration, links with traffic
TABLE VI

SIMULATION RESULTS FOR ASYMMETRIC TRAFFIC OVER RANDOM NETWORKS

\begin{tabular}{c|c|c|c|c}
\hline & $\begin{array}{c}\text { No. of obtained } \\
\text { solutions within } \\
100 \% \text { optimality }\end{array}$ & $\begin{array}{c}\text { No. of obtained } \\
\text { solutions with } \\
P \text { within } 10 \%\end{array}$ & $\begin{array}{c}\text { Average } \\
P\end{array}$ & $\begin{array}{c}\text { Average } \\
\text { runtime } \\
\text { second) }\end{array}$ \\
\hline \hline $\begin{array}{c}\text { Exhaustive } \\
\text { Search }\end{array}$ & 1,000 & 1,000 & $0 \%$ & 1.8511 \\
\hline HWF & 655 & 872 & $3.42 \%$ & 0.0017 \\
\hline MDF & 568 & 779 & $5.32 \%$ & 0.0019 \\
\hline
\end{tabular}

demands, 8, 9, and 10 have been chosen for scheduling. Then, we have an updated demand, $\mathbf{f}=[0,1,1,2,2,3,4,5,6,7]$ after this iteration. Now the traffic demands to be scheduled have a narrower range (i.e., 1 to 7 ) in the future. With compact traffic, in the later iterations, more scheduled links can be removed in each iteration because they have the same traffic demands. MDF, on the other hand, does not have such an advantage.

Additional simulations have further verified our observation. We have conducted five additional sets of simulations. Each set of simulations are based on different values of traffic demand range. The first set of simulations are based on $S_{1}(1 \sim 10)$, i.e., traffic demands randomly generated according to discrete uniform distribution with values ranging from 1 to 10 . The second set of simulations are based on $S_{2}(1 \sim 20)$ with values ranging from 1 to 20 , etc. For each set of simulations, we calculate the averaged $P$ values for HWF and MDF over 100 simulations. Fig. 12 plots the $P$ values versus different traffic ranges. It is shown in Fig. 12 that MDF and HWF perform comparably with $P$ of $3.54 \%$ and $3.52 \%$, respectively, when traffic demands ranges from 1 to 10 . However, $P$ of MDF increases quickly as the range of traffic demands increases, while HWF remains somewhat immune to such $P$ increase.

One possible improvement for future work is to integrate the two heuristic algorithms together. In particular, we could first sort the links according to their traffic demands in a descending order. Then, we schedule the links with the heaviest weight first. When there is a tie and two links have the same weight, we choose the link with the maximum degree in the associated conflict graph for scheduling. 


\section{CONCLUSiON}

In this paper, we have investigated MTR networks in which a node may simultaneously send to a number of other nodes; or simultaneously receive from other nodes. This capability can potentially improve the network capacity substantially. We have (i) provided a formal specification of MTR networks for a systematic study; (ii) formulated the link-scheduling problem of minimizing the airtime usage in an MTR wireless network as a linear program (LP) and demonstrated that it is NP-hard; (iii) proposed two computationally efficient heuristic algorithms to solve this LP; and (iv) presented extensive simulation results to show that both Heavy-Weight-First (HWF) and Maximum-Degree-First (MDF) algorithms achieve good optimality and runtime performance. With regard to (iv), both HWF and MDF have average percentage cost penalty less than $10 \%$ over 1,000 simulation experiments with different network topologies and traffic demands. The average runtime of both HWF and MDF is less than 0.01 second.

\section{REFERENCES}

[1] B. Raman and K. Chebrolu, "Revisiting MAC Design for an 802.11based Mesh Network," in ACM HotNets-III, 2004.

[2] R. Patra, S. Nedevschi, S. Surana, A. Sheth, L. Subramanian, and E. Brewer, "WiLDNet: Design and Implementation of High Performance WiFi Based Long Distance Networks," in USENIX NSDI, 2007.

[3] B. Raman and K. Chebrolu, "Design and Evaluation of a new MAC Protocol for Long-Distance 802.11 Mesh Networks," in ACM MobiCom, 2005.

[4] S. Surana, R. Patra, S. Nedevschi, M. Ramos, L. Subramanian, Y. BenDavid, and E. Brewer, "Beyond Pilots: Keeping Rural Wireless Networks Alive," in USENIX NSDI, 2008.

[5] S. Nedevschi, R. K. Patra, S. Surana, S. Ratnasamy, L. Subramanian, and E. A. Brewer, "An Adaptive, High Performance MAC for Long-distance Multihop Wireless Networks," in ACM MobiCom, 2008.

[6] S. Kakumanu and R. Sivakumar, "Glia: A practical solution for effective high datarate wifi-arrays," in Proc. of ACM MobiCom, 2009.

[7] B. Raman, "Channel Allocation in 802.11-Based Mesh Networks," in IEEE INFOCOM, 2006.

[8] L. Subramanian, S. Surana, R. Patra, S. Nedevschi, M. Ho, E. Brewer, and A. Sheth, "Rethinking Wireless for the Developing World," in $A C M$ SIGCOMM Hotnets-V, 2006.

[9] A. Sheth, S. Nedevschi, R. Patra, S. Surana, L. Subramanian, and E. Brewer, "Packet Loss Characterization in WiFi-based Long Distance Networks," in IEEE INFOCOM, 2007.

[10] S. Sen and B. Raman, "Long Distance Wireless Mesh Network Planning: Problem Formulation and Solution," in $W W W, 2007$.

[11] A. K. Das, R. J. Marks, P. Arabshahi, and A. Gray, "Power Controlled Minimum Frame Length Scheduling in TDMA Wireless Networks with Sectored Antennas," in IEEE INFOCOM, 2005.

[12] L. Fu, S. C. Liew, and J. Huang, "Joint Power Control and Link Scheduling in Wireless Networks for Throughput Optimization," in IEEE ICC, 2008.

[13] S. A. Borbash and A. Ephremides, "The Feasibility of Matchings in a Wireless Network," IEEE Transactions on Information Theory, vol. 52, no. 6, pp. 2749 - 2755, 2006.

[14] J. Tang, G. Xue, C. Chandler, and W. Zhang, "Link Scheduling with Power Control for Throughput Enhancement in Multihop Wireless Networks," IEEE Transactions on Vehicular Technology, vol. 5, no. 3, pp. 733 - 742, 2006.

[15] L. Fu, S. C. Liew, and J. Huang, "Power Controlled Scheduling with Consecutive Transmission Constraints: Complexity Analysis and Algorithm Design," in IEEE INFOCOM, 2009.

[16] S. A. Borbash and A. Ephremides, "Wireless Link Scheduling With Power Control and SINR Constraints," IEEE Transactions on Information Theory, vol. 52, no. 11, pp. 5106 - 5111, 2006.

[17] A. H. Land and A. G. Doig, "An Automatic Method for Solving Discrete Programming Problems,” Econometrica, vol. 28, pp. 497-520, 1960.

\section{APPENDIX A}

We consider the link-scheduling problem of finding the minimum frame length in an MTR network that has a bipartite graph structure. Not all networks can be casted into a bipartite structure, however. But for those network topologies that are bipartite graphs (including tree topologies), optimal scheduling is rather simple. Note that the term "graph" here refers to the structure of the network itself, rather than the conflict graph associated with the network.

A graph is bipartite if its vertex set can be partitioned into two subsets $A$ and $B$ so that each edge has one endpoint in $A$ and the other endpoint in $B$. Fig. 13 shows an example of a bipartite graph.

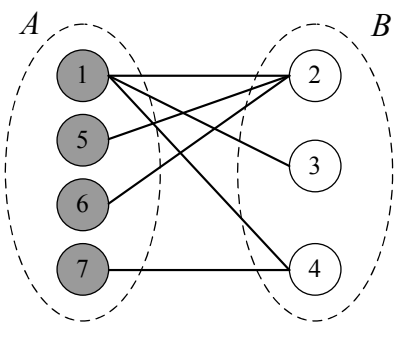

Fig. 13. The bipartite graph

A bipartite graph is a 2-colorable graph, i.e., we can use two colors to colorize all vertices in the graph. For example in Fig. 13, we can color all nodes $1,5,6$ and 7 in $A$ gray, and all node 2,3 and 4 in $B$ white. The gray nodes $1,5,6$ and 7 can operate in transmit mode while the white nodes 2,3 and 4 can operate in receive mode at the same time. Thus, MTR constraints can be easily met in a bipartite topology. Suppose the link set for the bipartite graph in Fig. 13 is $\{(1,2),(1,3),(1,4),(2,1),(2,5),(2,6),(3,1),(4,1),(4,7)$

$,(5,2),(6,2),(7,4)\}$ and the associated traffic demand vector is $\mathbf{f}=$ $[1,1,1,1,1,1,1,1,1,1,1,1,1,1]$. Then we only need two time slots to schedule all the traffic demands.

In general, if an MTR network is bipartite, we need $T_{\max }^{1}+T_{\max }^{2}$ time slots to fulfill all the traffic demands, where $T_{\max }^{1}$ is the maximum traffic demand among links in one direction in the bipartite graph; and $T_{\max }^{2}$ is the maximum traffic demand in the other direction.

Consider the bipartite example in Fig. 13 again. Suppose the traffic demand vector is $\mathbf{f}=[9,8,10,6,3,4,2,8,5,7,8,7]$. Then, to fulfill all the traffic demands, we can use $T_{\max }^{1}+T_{\max }^{2}=10+8=18$ time slots.

We can use a simple algorithm to solve the link scheduling problem in a bipartite MTR network. In the first step, we first choose the link with the maximum traffic demand $\left(T_{\max }^{1}\right)$ into the scheduling link set $E_{1}$. Then, we choose a link into link set $E_{1}$ if it does not conflict with the existing links in $E_{1}$ according the conflict graph. Repeat this process until no link can be added into link set $E_{1}$. We then assign $T_{\max }^{1}$ time slots to link set $E_{1}$. In the second step, we add all other remaining links into link set $E_{2}$. Then, we assign the maximum demand among all the remaining links, $T_{\max }^{2}$ time slots to link set $E_{2}$. Consider the above example again. The traffic demand vector is $\mathbf{f}=[9,8,10,6,3,4,2,8,5,7,8,7]$. Thus, in the first step, we have link set $E_{1}=(1,4),(1,2),(1,3),(5,2),(6,2),(7,4)$ and time slots $T_{\max }^{1}=10$ for link $(1,4)$. In the second step, we have link set $E_{2}=(2,1),(2,5),(2,6),(3,1),(4,1),(4,7)$ and time slots $T_{\max }^{2}=8$ for link $(4,1)$.

If the network is bipartite, we can easily solve the link scheduling problem in MTR networks. However, intentionally restricting the network topology to a bipartite graph may compromise network reliability and the network capacity [5]. Therefore, we need to consider more general topologies other than bipartite graphs. This is the motivation for our studies of more general network topologies in the main body of this paper. 


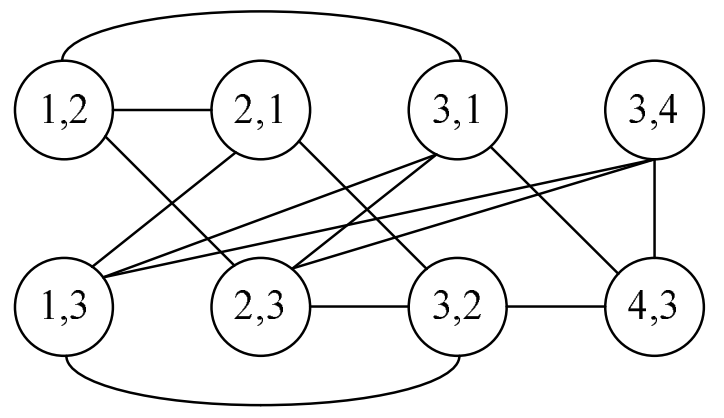




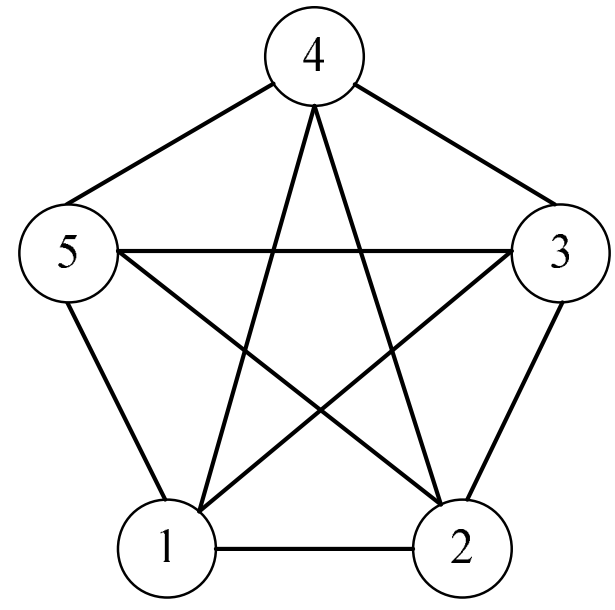




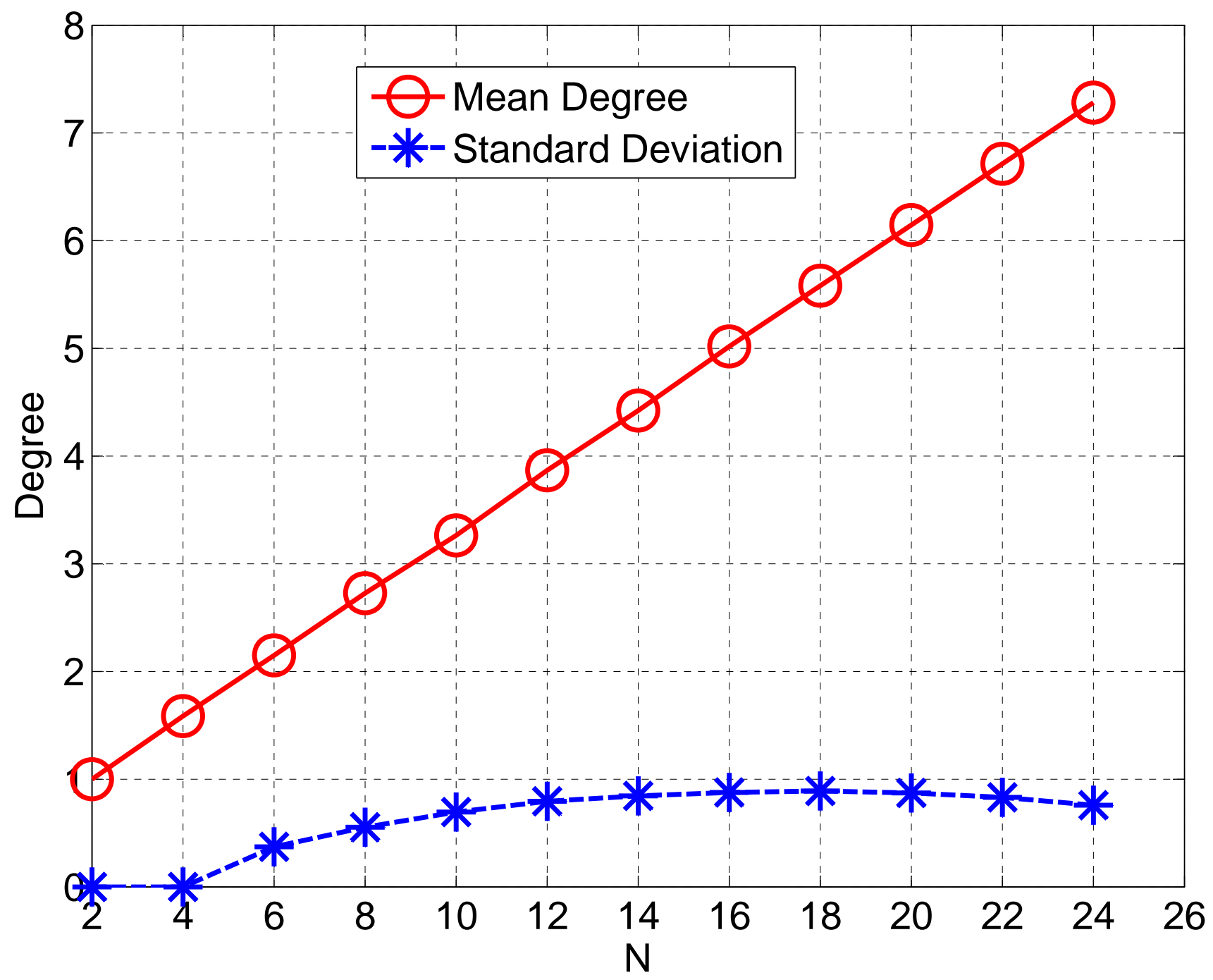




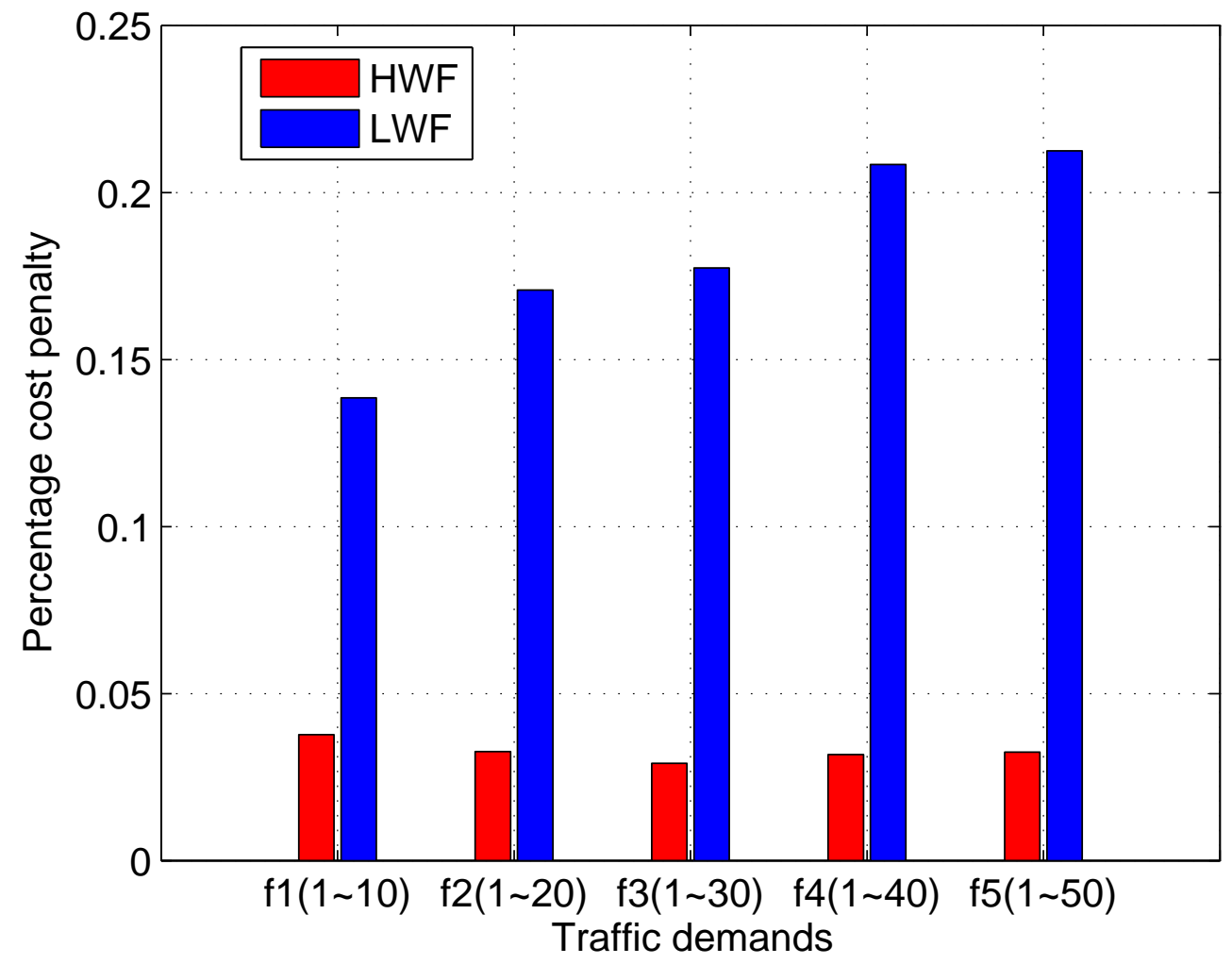




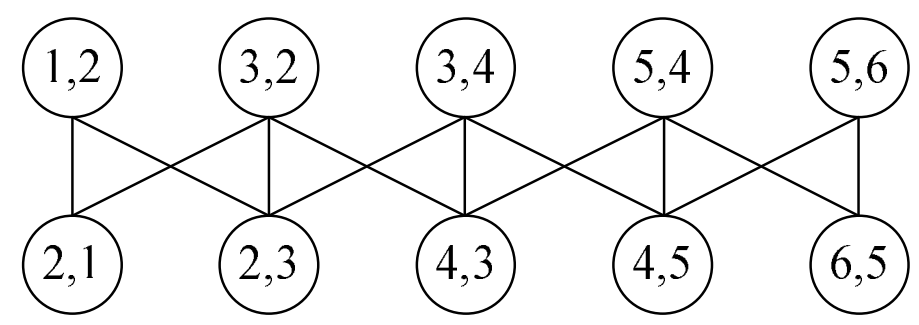




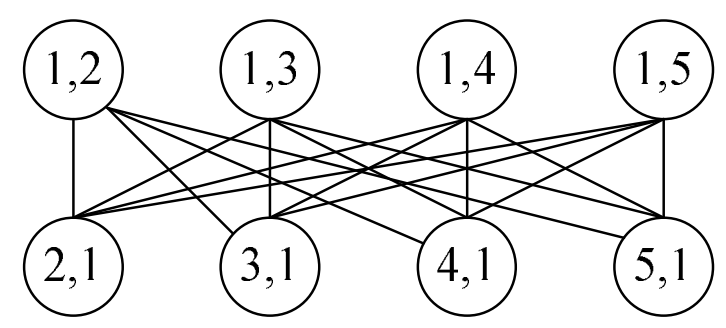




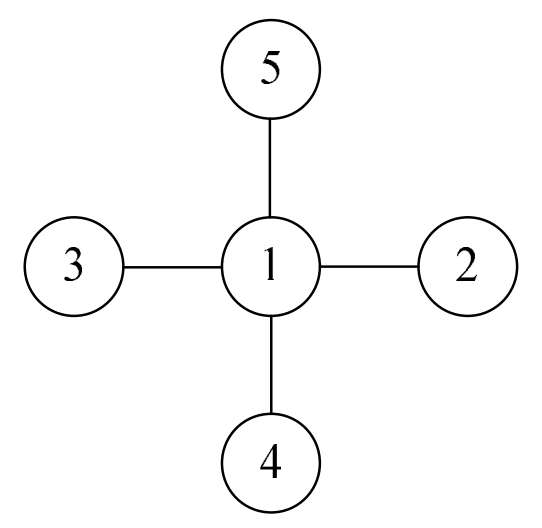




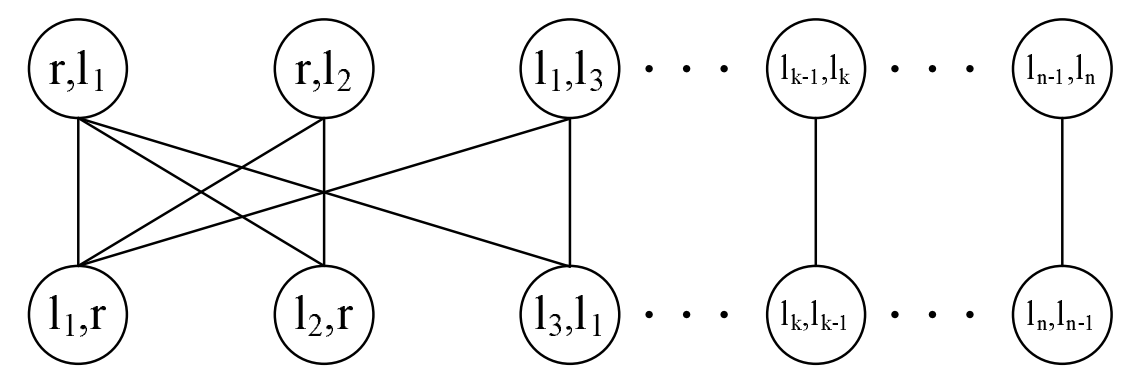




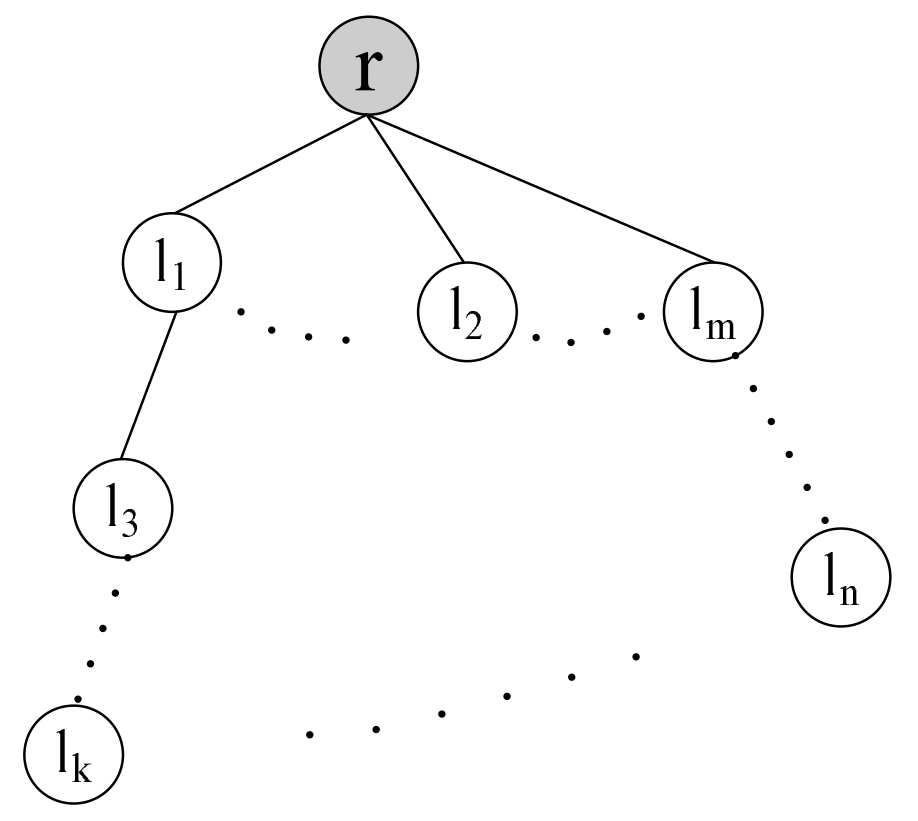

